\title{
Mutations in the human SC4MOL gene encoding a methyl sterol oxidase cause psoriasiform dermatitis, microcephaly, and developmental delay
}

\author{
Miao He,1,2 Lisa E. Kratz, ${ }^{3}$ Joshua J. Michel,2,4 Abbe N. Vallejo, ${ }^{2,4}$ Laura Ferris, ${ }^{5}$ \\ Richard I. Kelley, ${ }^{3}$ Jacqueline J. Hoover, ${ }^{2}$ Drazen Jukic, ${ }^{6}$ K. Michael Gibson, ${ }^{2,7}$ \\ Lynne A. Wolfe, ${ }^{2}$ Dhanya Ramachandran, ${ }^{1}$ Michael E. Zwick, ${ }^{1}$ and Jerry Vockley ${ }^{2,8}$ \\ 1Department of Human Genetics, Emory University School of Medicine, Atlanta, Georgia, USA. 2Department of Pediatrics, \\ University of Pittsburgh School of Medicine, Pittsburgh, Pennsylvania, USA. ${ }^{3}$ Kennedy Krieger Institute, Baltimore, Maryland, USA. \\ ${ }^{4}$ Department of Immunology, ${ }^{5}$ Department of Dermatology, and ${ }^{6}$ Department of Pathology, University of Pittsburgh School of Medicine, \\ Pittsburgh, Pennsylvania, USA. ${ }^{7}$ Department of Biological Sciences, Michigan Technological University, Houghton, Michigan, USA. \\ ${ }^{8}$ Department of Human Genetics, University of Pittsburgh Graduate School of Public Health, Pittsburgh, Pennsylvania, USA
}

\begin{abstract}
Defects in cholesterol synthesis result in a wide variety of symptoms, from neonatal lethality to the relatively mild dysmorphic features and developmental delay found in individuals with Smith-Lemli-Opitz syndrome. We report here the identification of mutations in sterol-C4-methyl oxidase-like gene (SC4MOL) as the cause of an autosomal recessive syndrome in a human patient with psoriasiform dermatitis, arthralgias, congenital cataracts, microcephaly, and developmental delay. This gene encodes a sterol-C4-methyl oxidase (SMO), which catalyzes demethylation of C4-methylsterols in the cholesterol synthesis pathway. C4-Methylsterols are meiosis-activating sterols (MASs). They exist at high concentrations in the testis and ovary and play roles in meiosis activation. In this study, we found that an accumulation of MASs in the patient led to cell overproliferation in both skin and blood. SMO deficiency also substantially altered immunocyte phenotype and in vitro function. MASs serve as ligands for liver $X$ receptors $\alpha$ and $\beta(L X R \alpha$ and $L X R \beta)$, which are important in regulating not only lipid transport in the epidermis, but also innate and adaptive immunity. Deficiency of SMO represents a biochemical defect in the cholesterol synthesis pathway, the clinical spectrum of which remains to be defined.
\end{abstract}

\section{Introduction}

Cholesterol is a key component of cell membranes and lipid rafts and is the immediate precursor of steroids, vitamin $\mathrm{D}$, and bile acids. Many disorders of cholesterol synthesis share common clinical features, such as abnormal morphogenesis, growth delay, and psychomotor disabilities (1). However, there are also striking differences suggesting that reduced de novo cholesterol synthesis per se may not primarily underlie some of the symptoms, including cataracts as well as skin and immune system abnormalities. Rather, recent studies implicate the accumulation of pre-cholesterol sterols and the replacement of cholesterol with some of these sterols in lipid rafts as playing a key role in the underlying pathophysiology (2). The meiosis-activating sterols (MASs) were the first group of cholesterol biogenesis intermediates that were found to have important extrahepatic functions in mammals. These include 4,4'-dimethyl-5 $\alpha$-cholesta-8,24-dien-3 $\beta$-ol (testis meiosis-activating sterol [T-MAS]), 4,4'-dimethyl-5 $\alpha$-cholesta8,14,24-trien-3 $\beta$-ol (follicular fluid meiosis-activating sterols [FF-MASs]), and zymosterol. They are found in high concentration in testis and ovary and play roles in oocyte maturation and meiosis activation. The function of the MASs outside the reproductive organs is not well studied. FF-MAS is also a ligand for liver $\mathrm{X}$ receptors (LXRs) (3). LXR signaling is known to regulate crosstalk between inflammatory and cholesterol metabolism,

Conflict of interest: The authors have declared that no conflict of interest exists. Citation for this article: J Clin Invest. 2011;121(3):976-984. doi:10.1172/JCI42650. which is important in regulating cholesterol efflux in epidermis (4). The association between the MASs and overproliferative inflammatory skin diseases is illustrated by a number of genetic defects in the pathway of processing of MASs. Six genes are expected to be involved in the metabolism of MASs (Supplemental Figure 3; supplemental material available online with this article; doi:10.1172/JCI42650DS1). Autosomal recessive defects in DHCR14B/LBR lead to hydrops-ectopic calcification-"motheaten" (HEM) dysplasia, which is a neonatal lethal condition in humans, while mutations in this gene in mouse cause ichthyosis and neutrophilic anomalies (5). Haploinsufficiency of DHCR14B/ $L B R$ leads to the Pelger-Huët anomaly of altered nuclear morphology in granulocytes (6). NSDHL and EBP are X-linked genes that lead to embryonic lethality in most males. Heterozygous mutations in NSDHL in females cause congenital hemidysplasia with ichthyosiform erythroderma and limb defects (CHILD) syndrome, and $E B P$ mutations cause chondrodysplasia punctata 2 (CDPX2). Mutations in the murine homologs of EBP and NSDHL lead to striated and bare patches on the skin (7).

In this study, we characterize genetic defects in SC4MOL (OMIM 607545), encoding a sterol-C4-methyl oxidase (SMO). This defect represents what we believe to be the first viable autosomal recessive condition in the MAS demethylation pathway, linking MAS demethylation with cell overproliferation, cholesterol homeostasis, and immune regulation in skin and blood and providing a model to study the pathogenesis of psoriasiform skin disease in this group of disorders. 



\section{Results}

Clinical presentation. A 13-year-old female presented for evaluation of severe ichthyosiform erythroderma affecting her entire body but sparing the palms (Figure 1, A-C). She did not show signs of dermatitis at birth; it was first noted around her umbilicus at the age of 2 and subsequently progressed to her back, trunk, and then the remainder of her body by the age of 6 . Her dermatitis worsened in the winter or when she was under stress, and once almost completely normalized. Also notable in the patient's medical history are congenital cataracts, mild developmental delay, microcephaly (head circumference $53.5 \mathrm{~cm}$ at the age of 13 years, <3rd percentile; 50 th percentile for a 7.5-year-old), and failure to thrive. Her height had increased along the 3 rd percentile between 9 and 39 months of age, and at age 13 years, her weight was $28.6 \mathrm{~kg}$ ( $<3 \mathrm{rd}$ percentile; 50 th percentile for a 9-year-old) and her height was $140 \mathrm{~cm}$ ( $<3 \mathrm{rd}$ percentile; 50th percentile for a 10.5-year-old). A skeletal survey showed delayed skeletal maturation, but bone morphology was normal. Skin histology showed psoriasiform hyperplasia, dilated capillaries in the dermal papillae, and neutrophils in the epidermis and stratum corneum (Figure 1D). Neutrophil elastase staining of neutrophils in the stratum corneum is shown in red in Figure 1F. Although this histology was initially considered to be consistent with psoriasiform dermatitis, more detailed examination of the tissue revealed the presence of several foamy cells in the dermis, and oil red $\mathrm{O}$ staining (Figure 1E) revealed intracellular lipid in these cells, reminiscent of that reported in patients with CHILD syndrome (8). However, the foamy cells were CD68 negative, indicating that they were not macrophages, a distinct difference from CHILD syndrome and verruciform xanthoma. Immunohistochemistry and H\&E-stained sections suggested that these cells were lipid-laden fibroblasts. Taken together, the results indicate that the histologic features are consistent with psoriasiform dermatitis with some features of a verruciform xanthoma (Figure 1, D and E). In light of the psoriasiform changes, traditional therapies for psoriasis were implemented for

\section{Figure 1}

Severe scaling and psoriasiform dermatitis in the patient. (A) Note mild microcephaly, lusterless, fine fair hair, and blepharitis. (B and C) The ichthyosiform erythroderma covers all of the patient's body except for the palms and soles. (D) H\&E-stained section of affected skin shows hyperkeratosis (original magnification, $\times 10$ ), loss of granular layer, psoriasiform hyperplasia, thinning of suprapapillary plate, and neutrophilic epidermal infiltration; these features are characteristic of psoriasis. (E) Oil red $O$ staining of affected skin biopsy (original magnification, $\times 20$ ) The arrow shows the intracellular lipid accumulation in the foamy cells in the dermis. (F) Neutrophil elastase staining (shown in red) of neutrophils in the stratum corneum of affected skin (original magnification, $\times 20)$. Photographs reproduced with signed informed consent/assent provided by the patient and her family.

the patient, including topical corticosteroids, calcipotriene, cyclosporine A, etanercept, phototherapy, and oral isotretinoin. However, while her skin briefly improved with cyclosporine A, she did not exhibit a long-term response to any of the therapies. Her serum lipid profile showed a persistently low total cholesterol level $(85 \mathrm{mg} / \mathrm{dl}$; normal, 140-176), low HDL (28 mg/dl; normal, 35-75), and low LDL (49 mg/dl; normal, 70-160) but normal triglycerides ( $84 \mathrm{mg} / \mathrm{dl}$; normal, 50-200) and VLDL $8 \mathrm{mg} / \mathrm{dl}$; normal, 8-14).

Biochemical and molecular characterization of SMO deficiency. A cholesterol biosynthesis defect was suspected on the basis of clinical presentation, and plasma sterols were analyzed by gas chromatography-mass spectrometry (GC-MS). The profile showed 20- and 500 -fold elevation of $4 \alpha$-monomethyl sterols and 4, 4'-dimethyl sterols, respectively (Figure 2, A-D). No 4-carboxylmethylsterol or 4-methylsterone was detected in either skin or plasma of the patient. To rule out the possibility that the patient's abnormal sterol pattern developed secondarily to another primary systemic process, we determined sterol profiles in extracts from cultured diploid skin fibroblast cultures from the patient and controls under normal culture condition and in cholesterol-deficient medium (Table 1 and Supplemental Table 4). Methylsterols were indeed markedly elevated in the patient's fibroblasts, but not any of the controls. Furthermore, the level of methylsterols in patient cells increased when they were grown in cholesterol-depleted medium. The sterol pattern in these experiments had not been previously associated with a human defect in the sterol synthesis pathway, but a review of the literature found it to be consistent with a defect in a methylsterol oxidase previously identified in yeast and plants $(9,10)$. Supplementation of culture medium with the addition of $1 \mu \mathrm{M}$ miconazole, a strong inhibitor of lanosterol $14 \alpha$ demethylase (CYP51, upstream of SMO) in cholesterol-deficient medium, and $5 \mu \mathrm{M}$ simvastatin, a compound that significantly reduces cholesterologenesis by inhibiting HMG-CoA reductase, reduced methylsterol levels in patient fibroblasts from $2 \%$ of total cholesterol to $0.9 \%$ and $0.5 \%$, respectively. 

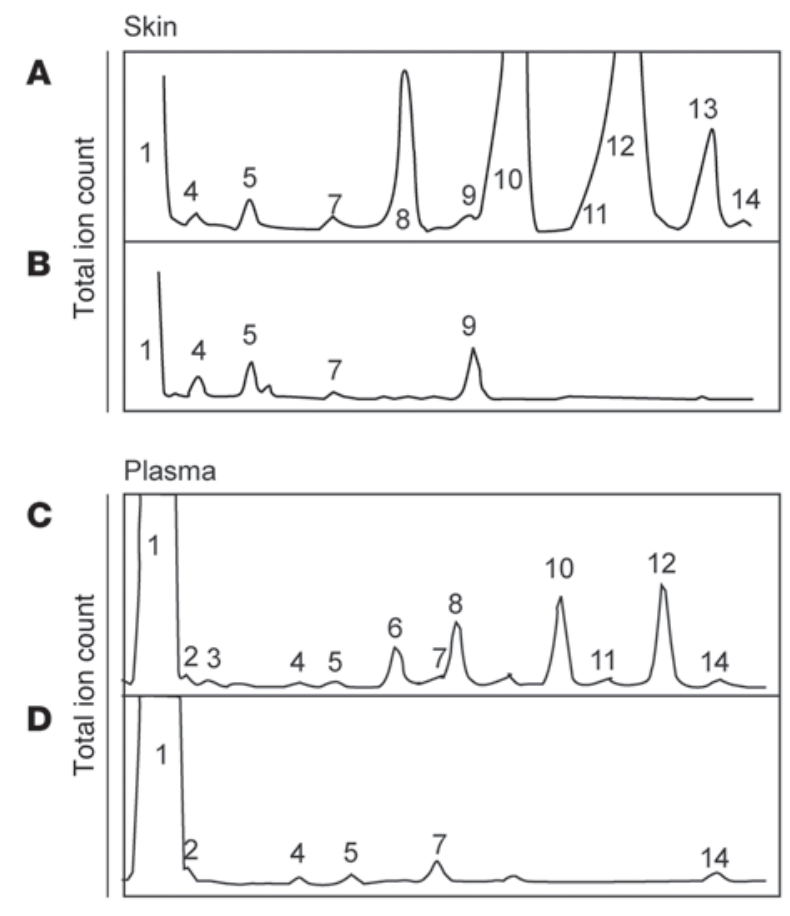

Retention time
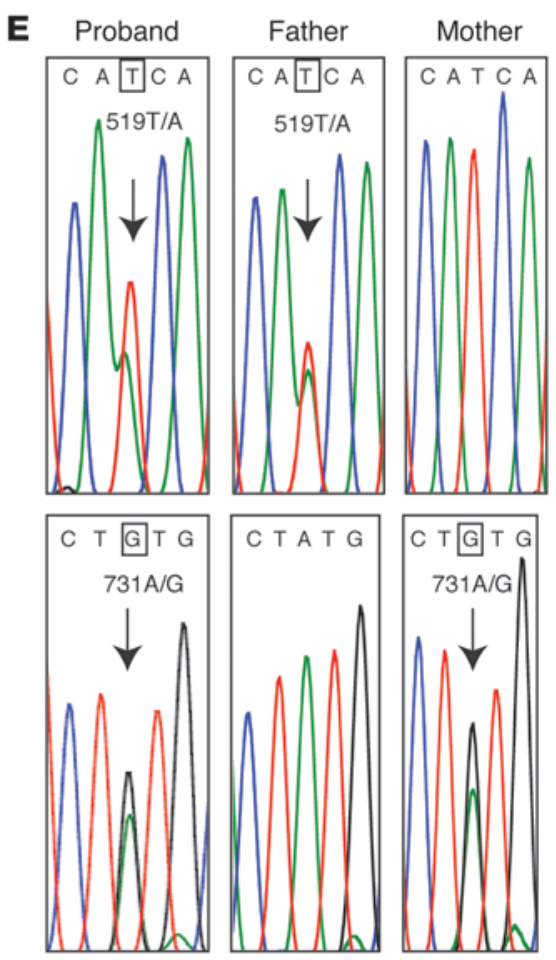

\section{Figure 2}

Sterol profiles of patient samples and mutation analysis of SC4MOL in proband and parental samples. GC-MS total ion current profiles of sterol extracts of patient skin (A), control skin (B), patient plasma (C), and control plasma (D). The ordinates are detector response, and the abscissas are elution time. The numbered compounds are: 1, cholesterol; 2, cholestanol; 3, 8(9)-cholestenol; 4, desmosterol plus 7-dehydrocholesterol;

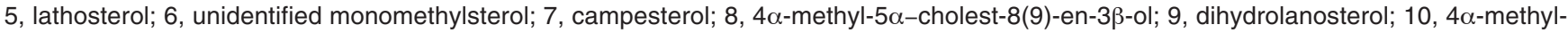
5a-cholest-7(8)-en-3 $\beta$-ol; 11 , unidentified isomer of 4,4'-dimethyl-5 $\alpha$-cholesta-8(9)-en-3 $\beta$-ol; $12,4,4$ '-dimethyl-5 $\alpha$-cholesta-8(9)-en-3 $\beta$-ol; 13 , $4,4^{\prime}$-dimethyl-5 $\alpha$-cholesta-8(9),24-dien-3 $\beta$-ol, 14 , sitosterol. The ion fragment patterns of each methyl sterol peak are shown in the Supplemental Note. Note that the column run for plasma elution time is slightly shifted compared with that of the skin samples. The levels of dimethylsterols and monomethylsterols were markedly increased in patient skin. Dimethylsterol (peak 6) is most elevated in skin, suggesting the preferential accumulation of 4,4'-dimethylsterols in the patient's skin. The absence of a 4-carboxysterol in the skin excludes a possible defect in NSDHL. (E) Two mutations were identified in SC4MOL from both gDNA and cDNA extracted from peripheral blood leukocytes: $519 T \rightarrow A$ (top) and 731A $\rightarrow$ G (bottom). 519T $\rightarrow$ A was also identified in gDNA from the patient's father, and $731 \mathrm{~A} \rightarrow \mathrm{G}$ was identified in the mother.

These data strongly indicated a block at the step of SMO in the cholesterol synthesis pathway. Since there is no validated enzyme assay or substrate available for SMO, we sequenced the SC4MOL gene predicted to encode this enzyme. Two variations from the published sequence (NM_006745) were identified: 519T $\rightarrow$ A and 731A $\rightarrow \mathrm{G}$ encoding H173Q and Y244C, respectively, in the SMO protein (Figure $2 \mathrm{E})$. The $519 \mathrm{~T} \rightarrow \mathrm{A}$ mutation was carried by the patient's father, and the $731 \mathrm{~A} \rightarrow \mathrm{G}$ mutation was present in the mother. Both positions encode amino acids that occur in highly conserved metal-binding domains in SMO (Supplemental Figure 1), and neither of the variants was identified in any of the databases for common SNPs. In addition, H173Q alters a predicted active site encompassing the second iron-binding motif of the enzyme. Both mutations are predicted to be "probably damaging" by the software package PolyPhen (http:// genetics.bwh.harvard.edu/pph/), with a score of 2.5 for the Y244C mutation and 3.2 for the H173Q mutation. Neither mutation was identified in 2,876 alleles from population controls. Nor were they present in the public SNP (http://www.ncbi.nlm.nih.gov/snp) or 1000 Genomes (http://browser.1000genomes.org/index.html) databases. Thus, these variants are exceptionally rare in the human population.
Because slightly elevated methylsterols have been reported in patients with CHILD syndrome (NSDHL deficiency), we also sequenced the cDNA from NSDHL gene, and no mutations were found.

Methylsterol levels were also increased (though less so) in the plasma of the patient's parents (Table 2), particularly the father, who carries the active site H173Q mutation, suggesting a subclinical effect in the heterozygous state. After 3 months of cholesterol supplementation, the patient's methylsterol level decreased by about $20 \%$, and the plasma cholesterol level was much improved, reaching a level near the low end of the normal range, but the methylsterol level decreased no further with longer treatment (Table 2).

Cell overproliferation in SMO deficiency. Previous studies of germinal cells have demonstrated meiosis-activating function of MASs, but the effect of MASs on cell cycle regulation and mitosis is unknown $(11,12)$. The fact that mutations in $D H C R 14 B / L B R$, $S C 4 M O L, N S D H L$, and EBP or its mouse homologs result in overproliferative skin disease prompted us to examine cell proliferation in skin fibroblasts from our patient (Figure 3, A-C). The rate of 
Table 1

Growth of patient and control fibroblasts on regular (10\% FBS) and cholesterol-depleted medium

\begin{tabular}{lcccc} 
& \multicolumn{2}{c}{ Control } & \multicolumn{2}{c}{ Patient } \\
& $\mathbf{1 0} \%$ FBS & $\begin{array}{c}\text { Cholesterol-depleted } \\
\mathbf{3 ~ d ~}\end{array}$ & $\mathbf{1 0 \%}$ FBS & $\begin{array}{c}\text { Cholesterol-depleted } \\
\mathbf{3 ~ d ~}\end{array}$ \\
Methylsterols $(\mu \mathrm{g} / \mathrm{mg}$ protein) & 2.3 & 1.4 & 12.3 & 24 \\
Total cholesterol $(\%)$ & 0.08 and $0.10^{\mathrm{A}}$ & 0.13 and 0.11 & 0.48 and 0.29 & 2.0 and 2.0 \\
Total protein $(\mathrm{mg})$ & 22 & 9.2 & 23 & 51
\end{tabular}

AData are from 1 experiment representative of 2 independent experiments. The total protein and cholesterol varied moderately between the 2 experiments, while the methylsterol in percent total cholesterol was consistent between replicates. The average methylsterol in control and patient cells was $0.12 \%$ and $2.0 \%$, respectively, in cholesterol-depleted medium; $0.09 \%$ and $0.4 \%$ in the regular medium.

cell division was found to be higher in patient fibroblasts than control cells when cultured in cholesterol-restricted medium, a condition under which de novo cholesterol biosynthesis is stimulated (Figure $3 \mathrm{~A}$ and ref. 13). Five different normal fibroblast lines with different passage numbers were used as controls. The S$\mathrm{G}_{2}-\mathrm{M}$ to $\mathrm{G}_{0}-\mathrm{G}_{1}$ ratio in patient skin fibroblasts was 3-fold higher than in control cells (Figure 3B). This ratio peaked after 2-3 days growth in the cholesterol-restricted medium and corresponded with peaks of cellular methylsterols and total protein (Table 2). Thus, cholesterol-restricted medium induced the patient cells to proceed into $\mathrm{S}-\mathrm{G}_{2}-\mathrm{M}$, implicating an association between MAS production and cell division. To further examine this possibility, we cultured transformed control human lymphoblasts in the presence of a sterol methyl oxidase inhibitor, 3-amino-1,2,4-triazole (ATZ) $(14,15)$. Under these conditions, the $S-G_{2}-M / G_{0}-G_{1}$ ratio increased 3 -fold in treated lymphoblasts, while neither simvastatin (an inhibitor of HMG-CoA reductase) nor fluconazole (a weak inhibitor of lanosterol $14 \alpha$ demethylase; CYP51) had a significant effect on cell cycle progression (Figure 3C). The stronger inhibitor miconazole was not used here due to its adverse effects on cell growth. These data strongly suggest that alterations in sterol methyl oxidase affect cell cycle activation.

Immune dysregulation in SMO deficiency. The patient was born with normal skin, and widespread skin lesions did not develop until she was 6 years old, suggesting that primary barrier dysfunction was unlikely to be the sole cause of her inflammatory skin condition. Many of the defects of cholesterologenesis are associated with immunological manifestations, and our patient's skin briefly improved with treatment with cyclosporine A; however, she subsequently became resistant to treatment, suggesting that her immune response was atypical compared with the other cholesterol pathway disorders and common psoriasis. We therefore examined a panel of immunologic parameters in the patient and her father as described in Methods and Supplemental Table 1. Blood samples from 20 normal individuals were studied as controls. Selected multicolor flow cytometry profiles of granulocytes and $\mathrm{T}$ cells are shown in Figure 4A, and the geometric mean fluorescence intensity (GMFI) and percentage of cells for each measurement are shown in Supplemental Table 2. We found that activated $\mathrm{CD} 16^{+}$granulocytes (identified by $\mathrm{CD} 25^{+} \mathrm{CD} 69^{+}$and $\mathrm{CD} 86^{+} \mathrm{HLA}-$ $\mathrm{DR}^{+}$subsets) were increased 30- and 20-fold in the patient and her father, respectively, compared with healthy controls (Figure 4A and Supplemental Table 2). CD86 and HLA-DR are markers for antigen-presenting cells. Interestingly, it has been reported that blocking de novo cholesterol synthesis with a statin can prevent these markers from expressing in dedicated antigen-presenting cells (16). In addition, there were 30 - and 15 -fold increases in the numbers of TLR-2 ${ }^{+}$TLR-4- granulocytes in the patient and her father, respectively, compared with healthy controls (Figure 4A). Upregulation of TLR-2, but not TLR-4, is considered typical for patients with psoriasis (17) or psoriatic arthritis (18), but not those with rheumatoid arthritis. Overexpression of TLR-2 and downregulation of TLR-4 in granulocytes likely reflects dysregulation of the immune response in the patient following normal bacterial infections. Consistent with this interpretation, expression of the granulocyte-specific CD16b isoform was also markedly downregulated in both the patient and her father (Figure 4B), suggesting a defect in phagocytic function. No significant differences were observed in the monocyte population (data not shown). In the lymphocyte compartment, both patient and father had a significantly higher proportion of CD8 ${ }^{\mathrm{dim}} \mathrm{T}$ cells that were also $\mathrm{CD}^{2} 8^{-} \mathrm{CD} 56^{+}$compared with controls (Figure 4A and Supplemental Table 2). Downregulation of CD8 and CD28 and accumulations of $\mathrm{CD} 28^{-} \mathrm{CD} 56^{+} \mathrm{T}$ cells are indicative of pervasive immune activation in the setting of chronic inflammatory disease or aging (19). It is surprising that the patient's father, who has normal skin, showed an immunologic profile similar to that of the patient, and that both are strikingly different from all the controls in this study and those seen with other inflammatory diseases (19).

Because psoriasis and psoriatic arthritis are known to be driven by the production of certain cytokines, including the TNF- $\alpha$ and IL-6, the serum cytokine profiles of the patient and father were investigated by multiplex Luminex assay (Supplemental Table 3). Consistent with the flow cytometry data, the levels of a number of proinflammatory and chemokines associated with granulocyte and monocyte

\section{Table 2}

Plasma sterol analysis of controls; patient before cholesterol supplementation; patient after 3 months of cholesterol supplementation; and the patient's mother and father

\begin{tabular}{lcc}
\hline & $\begin{array}{c}\text { Methylsterols } \\
(\mathbf{m g} / \mathbf{m l})\end{array}$ & $\begin{array}{c}\text { Total cholesterol } \\
(\mathbf{m g} / \mathbf{d l})\end{array}$ \\
Normal & $2.8-3.2$ & $140-176$ \\
Patient & 41 and $42^{\mathrm{A}}$ & 85 and $93^{\mathrm{A}}$ \\
Patient + cholesterol & $32 \pm 1.7^{\mathrm{B}}$ & $117 \pm 12$ \\
Mother & 6.4 & 188 \\
Father & 18.5 & 231
\end{tabular}

ABaseline methylsterol and cholesterol levels in patient are from measurements at 2 time points that were 1 month apart. BMean \pm SD of measurement at three time points: 1,3 , and 5 months after start of treatment. 

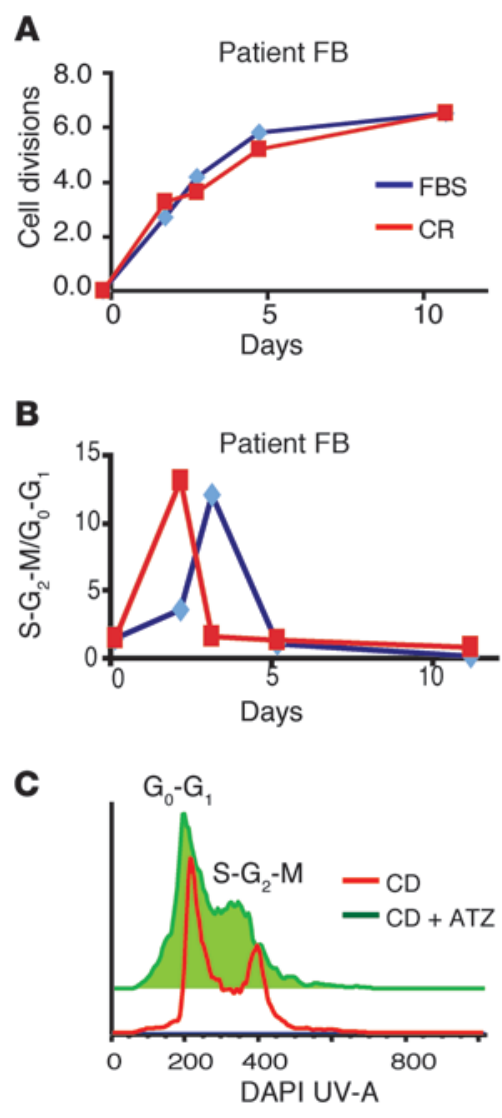

\section{Figure 3}

Cell proliferation and cycle abnormalities in SMO deficiency. (A and B) Fibroblasts were labeled with CFSE and DAPI and analyzed by flow cytometry. The number of cell divisions was determined by the number of CFSE fluorescence peaks. The number of cells in the $\mathrm{G}_{0}-\mathrm{G}_{1}$ and $\mathrm{S}-\mathrm{G}_{2}-\mathrm{M}$ phases of the cell cycle was analyzed by the amount of linear DAPI fluorescence. (C) Histograms show typical DAPI fluorescence profiles of cells in $\mathrm{G}_{0}-\mathrm{G}_{1}$ and $\mathrm{G}_{2}-\mathrm{S}-\mathrm{M}$ phases of the cell cycle in normal human EBV-transformed lymphoblasts cultured in standard medium with $10 \%$ FBS; in cholesterol-depleted medium (CD); or in CD supplemented with either simvastatin (ST), ATZ, or fluconazole (FA). The control data represent 5 different normal cell lines with different passages. The degree of decrease in cell divisions was similar in all the controls when growing in cholesterol-depleted medium compared with regular medium. Fluorescence is expressed as GMFI. All GMFI values shown have robust coefficients of variation less than $1 \%$. activation were increased in the patient, and to a lesser extent in her father, including GM-CSF, a cytokine reported to trigger psoriasiform eruption in humans (20). IL-6 and IL-8 were also elevated in the patient's serum (Supplemental Table 3). Elevation of IL-6 and IL-8 has been demonstrated in psoriatic skin and fibroblasts and is considered an amplifier of skin inflammation (21). The classical proinflammatory cytokine TNF- $\alpha$ was normal in the patient, consistent with the failure of the dermatitis to respond to treatment with TNF$\alpha$ inhibitor etanercept. Cultures of patient skin fibroblasts showed constitutive production of IL- 6 compared with control fibroblasts regardless of passage number (Figure 4D), and IL- 6 concentration was further increased with the addition of recombinant TNF- $\alpha$, a known inducer of IL-6. In agreement with these findings, statins have been reported to suppress Th1-biased cytokine production in both cultured microglia and a mouse model (22).

To investigate a possible association between the defect in cholesterologenesis and cytokine production, we treated control and patient fibroblasts with simvastatin, which reduces cholesterol biosynthesis intermediates including methylsterols (23, 24). After 24 hours, IL- 6 production by patient fibroblasts was significantly decreased even in the presence of exogenous TNF- $\alpha$ (Figure 4D), suggesting that the abnormal cytokine production is likely associated with cholesterologenesis.

Reduction of TLR-4 expression induced by SMO inbibition. Activation of TLRs such as TLR4 by microbial ligands inhibits cholesterol efflux by regulating $\mathrm{ABCA} 1$ (4), promoting immunocyte proliferation through LXR signaling (25). Of note, ABCA1 is an important transporter for controlling lipid and cholesterol composition in epidermis, critical to maintenance of normal skin barrier function (26). Therefore, we next examined expression of TLR receptors by direct inhibition of SMO in normal leukocytes. Strikingly, by inhibiting SMO in normal leukocytes with ATZ, we found a greater than 6-fold increase in the TLR-2 ${ }^{+}$TLR-4- ${ }^{-}$granulocyte population (Figure 4C) and 2-fold decrease in CD8 expression in normal lymphocytes (Table 3 ). Half of the TLR-2-TLR- $4^{+}$and $40 \%$ of TLR- $2^{+}$TLR $-4^{+}$normal granulocytes lost their TLR-4 expression, and the majority of them became TLR2 positive, suggesting a direct regulating effect of sterol methyl oxidase on TLR receptor expression. TLRs are the connection between innate and adaptive immunities in humans and also the connection between bacterial infection and lipid metabolism in skin.

\section{Discussion}

Sterols are important components of cell membranes and lipid rafts, and serve as precursors of many bioactive compounds. SC4MOL is expressed almost ubiquitously in human tissues according to EST database profiles (http://www.ncbi.nlm.nih. gov/UniGene/ESTProfileViewer.cgi?uglist=Hs.105269) and the gene atlas of the human protein-encoding transcriptome in 79 different human tissues from a variety of sources in the GEO database (GDS596; http://www.ncbi.nlm.nih.gov/sites/GDSbrowser). The expression of this gene is highest in liver, brain, adrenal gland, lymphoblast, skin, testis, and vascular tissues. Expression data of SC4MOL in cultured epidermal keratinocytes (GDS2611, GDS2903) and normal leukocytes (GDS3207) can also be found in the GEO database.

A C4 demethylation reaction is found in plants, animals, and yeast, although the structure of sterols and their biosynthetic pathways vary in each group. Previous studies have demonstrated that 
A All Leukocytes
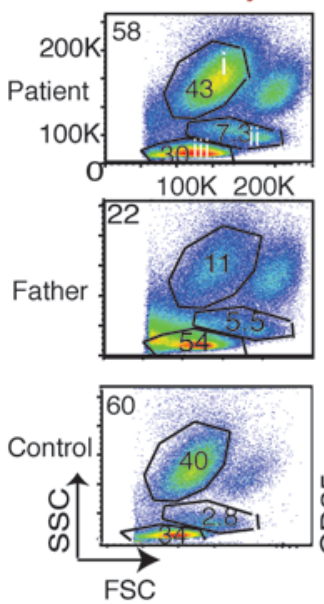

B

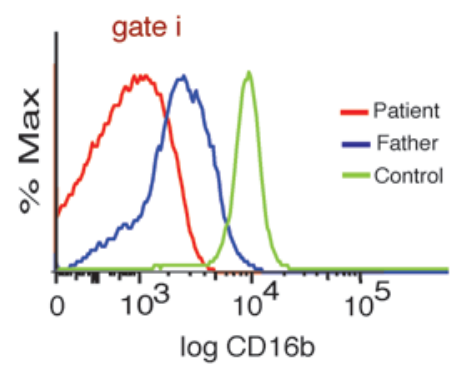

A: Granulocytes
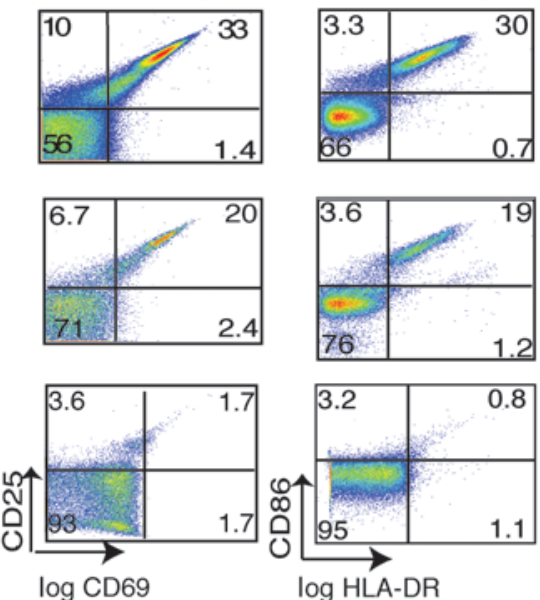

$\log$ CD69

A: Granulocytes
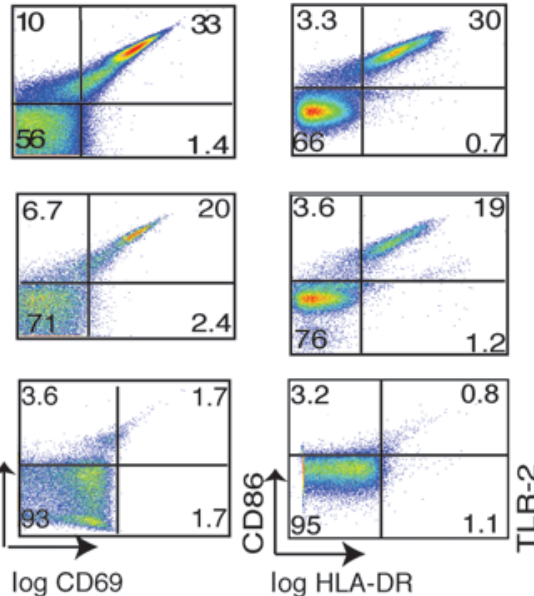

log HLA-DR
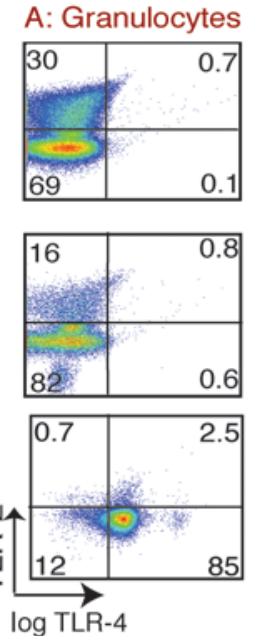
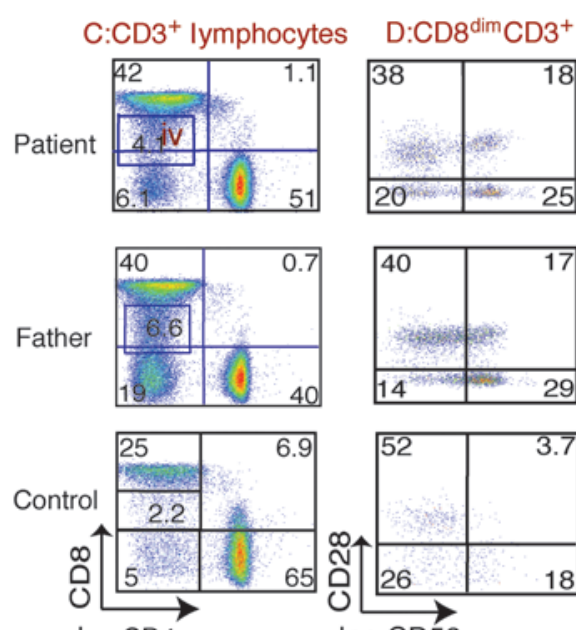

$\log \mathrm{CD} 4$

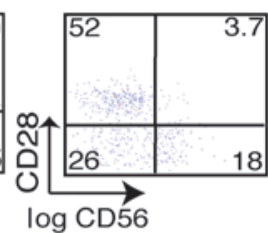

D

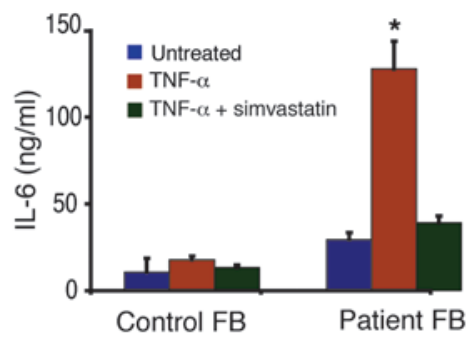

Figure 4

Immunocyte abnormalities in SMO deficiency. (A and B) Leukocyte populations were analyzed by multicolor flow cytometry; subsets identified by forward/scatter profiles, with mature granulocytes, monocytes, and lymphocytes falling in gates $\mathrm{A}$, B, and C, respectively. The control data shown here represent blood samples donated from 20 normal individuals. (A) Cytometric profiles for CD25, CD69, CD86, HLA-DR, TLR-2, and TLR-4 (gate i, and fluorescence histograms of CD16b) and (B) for mature granulocytes/neutrophils. Also shown in A are the CD4, CD8 profiles of $\mathrm{CD}^{+} \mathrm{T}$ cells; and CD28 ${ }^{\text {null }} \mathrm{CD} 56^{+}$in CD8 ${ }^{\text {dim }} \mathrm{T}$ cells (gate iv). No significant differences were observed in the monocyte population (data not shown). (C) Overlay of treated and untreated cytometric profiles. Note that $50 \%$ of control granulocytes migrated to TLR-2+TLR-4- after the ATZ treatment. (D) IL-6 production by skin fibroblasts from healthy control and patient upon treatment of TNF- $\alpha$, and TNF- $\alpha$ plus simvastatin, or in medium alone. ${ }^{\star} P<0.01$. Data are presented as mean \pm SD.

only one SMO in yeast is involved in demethylation of both $4,4^{\prime}$ dimethyl and $4 \alpha$-monomethyl sterols, while two different SMOs exist in plants (9). Since there is only one known SMO in humans (encoded by the SC4MOL gene), we speculate that human SMO will function similarly to its yeast homolog. The sterol intermediates detected in our SMO-deficient patient for the first time to our knowledge provide direct evidence of the role of human SMO in demethylation of 4,4'-dimethyl and $4 \alpha$-monomethylsterols. Besides the accumulation of 4,4'-dimethylsterol, the patient accumulated a significantly increased amount of $4 \alpha$-monomethylsterols, which suggests that both 4,4'-dimethylsterols and $4 \alpha$-monomethylsterols are substrates for human SMO. No 3-oxo-4 $\alpha$-methylsterols were detected in either plasma or skin of the patient, suggesting a functional difference between human and yeast SMO (27). Thus, it is unlikely that human SMO utilizes 3-oxomethylsterol as a substrate. Rather, in contrast to yeast, 3 -oxomethylsterol is reduced to $4 \alpha$ monomethylsterol by 3-ketosteroid reductase (HSD17B7) before it enters the second round of demethylation in humans (Supplemental Figure 3 and ref. 28). In addition, although 4,4'-dimethylsterol was more abundant than $4 \alpha$-monomethylsterols in the samples from our patient, only $4 \alpha$-monomethyl-7-en-sterol accumulated, with no trace of 4,4'-dimethyl-7-en-sterol. These findings suggest that $4 \alpha$-monomethylsterol, but not the 4,4'-dimethylsterols, can be utilized downstream by $\Delta 8-\Delta 7$ isomerase. Taken together, this pattern indicates that $4 \alpha$-monomethylsterol may have physiological functions differing from those of 4,4'-dimethylsterol.

Skin cell hyperproliferation causes the flaking skin and characteristic histopathology seen in psoriasiform dermatitis. All known genetic disorders that cause increased levels of MASs present with ichthyosiform or psoriasiform dermatitis in humans and mice, including defects in DHCR14/LBR, NSDHL, and EBP (Supplemental Figure 3 and refs. 1, 5, 7). However, none of the defects adjacent or distal to the MAS demethylation complex, including CYP51, $S C 5 D$, or $D H C R 7$, result in skin lesions $(24,29,30)$. The present cell proliferation studies revealed a perturbation of cell cycle in cells from our patient, both in cholesterol-restricted medium (which induces increased de novo cholesterol biosynthesis) and with specific inhibition of SMO by ATZ. Interestingly, patients with genetic 


\section{Table 3}

Analysis of granulocyte and T cell phenotypes of normal leukocytes treated with ATZ by multicolor flow cytometry

\begin{tabular}{|c|c|c|}
\hline Cell population & Untreated & ATZ-treated ${ }^{A}$ \\
\hline 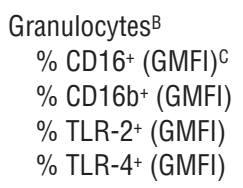 & $\begin{array}{c}80.6(57) \\
89.4(48.2) \\
45.2(129) \\
85.0(67.8)\end{array}$ & $\begin{array}{l}60.7(55.6) \\
61.2(40.3) \\
68.0(397) \\
46.0(35.8)\end{array}$ \\
\hline $\begin{array}{l}\mathrm{CD}^{+} \mathrm{T}^{\mathrm{T}} \text { cells } \\
\% \mathrm{CD}^{+}(\mathrm{GMFI})\end{array}$ & 26.7 (38.9) & $27.7(26.1)$ \\
\hline
\end{tabular}

${ }^{A} A T Z$ treatment had no significant effect on cell viability as determined by trypan blue exclusion. ${ }^{B}$ Granulocyte gate i in Figure 4D; CD3-CD20$\mathrm{CD}^{2} 6^{+}$cells. CGMFI of the indicated antigenic marker; all GMFI values shown have robust coefficients of variation less than $1 \%$. DLymphocyte gate iii in Figure 4D, and gated for CD $3^{+} \mathrm{CD} 20-\mathrm{CD} 16-\mathrm{CD} 8^{+}$cells.

defects in NSDHL, which encodes the enzyme immediately downstream of SMO, occasionally present with clinical features nearly identical to those in individuals with defects in EBP; however, they differ in the histopathology of their skin lesions (1). Outside of the newborn period, NSDHL-deficient patients have psoriasiform dermatitis, and sometimes verruciform xanthoma-like lesions secondary to the presence of lipid-laden macrophages in the dermis. $E B P$-deficient individuals have early resolution of their skin disease, with a typical appearance of atrophy but not inflammation on skin biopsy. We have previously reported the presence of elevated methylsterols in skin flakes from patients with common psoriasis (31). Our patient and NSDHL-deficient patients accumulate 4,4'-dimethylsterols in the skin, but EBP-deficient patients do not. This implies that although the MASs may play a role in overproliferation of skin cells and disruption of skin barrier function, 4,4'-dimethylsterols are unique in contributing to perturbation of immune function.

Almost all of the genetic diseases in cholesterol biosynthesis present with some degree of immune dysfunction, including SmithLemli-Opitz syndrome (SLOS) and mevalonate kinase (MVK) deficiency $(32,33)$. Patients with mild mutations in the MVK gene present with hyper-IgD syndrome, periodic fever, and high levels of IgA. Skin lesions, arthritis, lymphadenopathy, and splenomegaly are common in these patients. Previous studies suggest that anomalies in the immune system of SLOS and MVK patients may relate to the accumulation of mevalonate and associated isoprenoid synthesis (34) or abnormal cholesterol composition of lipid rafts in immunocytes (35). However, these studies did not report comprehensive immunological parameters or immunocyte profiles. Thus, our results provide the first evidence to our knowledge that the immunologic abnormalities seen in patients with defects in cholesterologenesis are distinct from other disorders. Since epidermal lipid composition is important for skin barrier function, a defect in de novo cholesterol synthesis that affects epidermal lipid composition could impair barrier function and contribute to dysregulation of immune function. However, the unique immunocyte profile seen in our patient and her father is strikingly different from that usually seen with skin barrier dysfunction, suggesting they are unlikely solely secondary abnormalities. In addition, methylsterols are potent ligands for LXR, while most other sterols are not, and activated LXR binds to TLR-4 promoter through an LXRE site to regulate TLR-4 expression (36). Thus, the reduction of TLR-4 expression in SMO deficiency may be related to inhibition of LXR by T-MAS, which is structural similar to FF-MAS. Since TLR-4 plays an inhibitory role in both immune response and sterol efflux by ABCA1, the reduction in TLR4 expression could lead to increased lipid transport and cytokine response in the skin in response to bacterial infection, ultimately resulting in the severe psoriasiform dermatitis seen in our patient. Interestingly, the promoter region of ABCA1 also has an LXRE site, which is strongly inhibited by TLR-4 signaling (4). These potential connections imply the unique functions of sterol methyl oxidase in immune function regulation in skin and blood.

Of note, the most profound immunocyte abnormalities in the patient and, to a lesser degree, in her father were found in the granulocytes. Defects in forming mature granulocyte nuclei are also seen in individuals who carry a C14 sterol reductase (lamin $\mathrm{B}$ receptor) mutation (37). The appearance of similar granulocyte abnormalities in individuals with defects in adjacent steps of the cholesterol synthesis pathway could be coincidental. However, granulocyte/neutrophil-rich pathology is also characteristic of psoriasis and psoriatic arthritis, and our findings suggest a direct association between demethylation of MASs and innate immunity $(38,39)$. It is also intriguing to speculate that the mild elevation of 4,4'-dimethylsterols seen in the patient's father could be relevant to his history of early-onset inflammatory joint disease. In support of this, two other paternal relatives with histories of early-onset arthritis also carry the $519 \mathrm{~T} \rightarrow \mathrm{A}$ mutation found in the father (Supplemental Figure 2). Interestingly, it has become evident in recent years that statins suppresses several key functions of the immune system and influence the development of autoimmune disease independent of cholesterol reduction (40). Rather, its immune-modulating effects are likely related to the reduction in cholesterologenesis intermediates (41).

In summary, we have identified the first patient to our knowledge deficient in SMO, a novel enzyme in the C4 methylsterol demethylation complex. She had congenital cataracts and developed psoriasiform dermatitis with the presence of lipid-laden cells in the dermis in a pattern reminiscent of CHILD syndrome. Importantly, our patient also had an aberrant TLR expression profile likely induced by the abnormal metabolites accumulating due to the SMO deficiency. Our studies provide evidence that SC4MOL gene function not only is important in de novo sterol synthesis and thus critical for skin barrier function (42), but also regulates cell proliferation and immune regulation. Taken together, the results indicate that defects in SC4MOL gene affect most of the major processes that are critical to the pathogenesis of psoriasiform dermatitis.

\section{Methods}

This study was conducted according to an IRB protocol approved by the University of Pittsburgh. Written informed consent was received from participants in the study.

Genomic DNA and cDNA amplification and sequencing. Total RNA and genomic DNA (gDNA) were extracted from patient fibroblasts for reverse transcription, PCR amplification, and sequencing as described previously (43). Primers 5'CTTTTACCTGAGAATCCTCTGG-3' (forward) and 5'-GCAGAGGATTCTCAGGTAAAAG-3' (reverse) were used for cDNA amplification covering the entire coding region. Primers 5'-ACTTTCTAATCTCTACTGTTCAGCAC-3' (forward) and 5'GAATGATGGAAGAGACGGTA-3' (reverse) were used for the PCR amplification of exon 6 to confirm the $731 \mathrm{~A} \rightarrow \mathrm{G}$ (Y224C) variant, and primer 5'-GGTCAACCTGGTAAGTGG-3' (forward) and primer 5'-TGTTTGTATGTGTGTGTATTG-3' (reverse) were used for the PCR amplification of exon 4 to confirm the $519 \mathrm{~T} \rightarrow \mathrm{A}(\mathrm{H} 173 \mathrm{Q})$ variant. 
TaqMan genotyping. Control samples used for the genotyping of novel variants were obtained from the National Institute of Mental Health Human Genetics Initiative. All controls (1,452 samples) were adult males of European descent who had been screened to rule out psychiatric disorders. In addition, known positive controls and negative water controls were also included in each plate assayed. Genotyping was performed by a custom TaqMan SNP genotyping assay from Applied Biosystems as per the manufacturer's instructions. The primers and probes were designed according to the Custom TaqMan SNP Genotyping Assay Protocol (Applied Biosystems). For the 519T $\rightarrow$ A (H173Q) variant, amplification primers used were 5'-TCTGCATAGACTCTTACACCACAAAAG-3' (forward) and 5'-GCCTCTAACAGAAAGAATTAAATATAACTCTCACA-3' (reverse). Allele-specific fluorophore-labeled reporter probes used included 5'-CTGAAACTCATGATGAACTT-3' (VIC) and 5'-TGAAACTCATGTTGAACTT-3' (FAM). For the 731A $\rightarrow$ G (Y224C) variant, amplification primers used were $5^{\prime}$-ATATTCCTCTCAACCCTTTAAATCTGATCC-3' (forward) and 5'-TCCAATGAAGTTCATGTGGTGGAAA-3' (reverse). Allele-specific fluorophore-labeled reporter probes used included 5'-AGAACCAGCATAGAAAG-3' (VIC) and 5'-AACCAGCACAGAAAG-3' (FAM). Assays were performed in 384-well plates, and the final volume of the reaction was $5 \mu \mathrm{l}$, consisting of $5 \mathrm{ng}$ of genomic DNA, $2.5 \mu \mathrm{l}$ of TaqMan Master Mix, and $0.06 \mu \mathrm{l}$ of $40 \times$ assay mix. Thermal cycle conditions were $50^{\circ} \mathrm{C}$ for 2 minutes, $95^{\circ} \mathrm{C}$ for 10 minutes, and 45 cycles of $92^{\circ} \mathrm{C}$ for 15 seconds and $58^{\circ} \mathrm{C}$ for 1 minute. Upon completion of PCR, plates were read on an ABI PRISM 7900 HT sequence detector. Genotypes were called successfully for 1,438 samples at both variants using Allelic Discrimination Sequence Detection Software (Applied Biosystems) version 2.3 (SDS v2.3).

Sterol analyses. Sterol distribution was measured in cultured skin fibroblasts, transformed lymphocytes, serum, and skin flakes obtained from patients and controls by gas chromatography and selected-ion mass spectrometry as described previously $(7,44)$.

Flow cytometry on peripheral blood cells. Flow cytometry on cells from patients and controls was carried out and analyzed as described previously (19). In the sterol oxidase inhibition studies, $10 \mathrm{ml}$ red blood cell-depleted whole blood from a healthy 16-year-old girl were incubated with $5 \mathrm{mM} \mathrm{ATZ} \mathrm{for} 60$ hours at $37^{\circ} \mathrm{C}$ with $5 \% \mathrm{CO}_{2}$. Both ATZ-treated and untreated total leukocytes were then isolated and stained as described in Supplemental Table 2.

Serum cytokine quantification. Cytokine levels were determined by multiplex assay using the Luminex system (Luminex Corp.) as described previously $(45,46)$.

Cell culture, proliferation, and ELISA. Primary dermal fibroblasts were cultured in DMEM supplemented with $10 \% \mathrm{FBS}, 100 \mathrm{U} / \mathrm{ml}$ penicillin, and $100 \mathrm{mg} / \mathrm{ml}$ streptomycin; GlutaMAX (Invitrogen), TNF- $\alpha$ (10 ng/ml), and simvastatin $(5 \mu \mathrm{M})$ were added to fibroblasts at $80 \%$ confluence; and culture media were collected after 24 hours for measurement of IL-6 using an ELISA kit (BD). For cell proliferation assays, primary fibroblasts or EBV-transformed human lymphoblasts were cultured with cholesterol-depleted medium as described
(44), in which condition de novo synthesis of cholesterol was activated and maintained (Supplemental Table 5); and ATZ (2 mM), simvastatin $(5 \mu \mathrm{M})$, or fluconazole $(12.5 \mu \mathrm{M})$ was added to the medium before the cells were subjected to the proliferation assay.

Cell proliferation and cell cycle stage analysis. Skin fibroblasts or EBV-transformed lymphoblast $\left(2 \times 10^{7}\right.$ cells $\left./ \mathrm{ml}\right)$ were labeled with 5- (and 6-)CFSE $(0.25 \mu \mathrm{M})$ (Molecular Probes, Invitrogen) using established procedures (19). At different days of culture, cells were harvested and stained with DAPI. The fluorescence intensity of both CFSE and DAPI was analyzed by multicolor flow cytometry. The number of cell divisions was determined by the number of CFSE fluorescence peaks. The number of cells in $\mathrm{G}_{0}-\mathrm{G}_{1}$ and $\mathrm{S}-\mathrm{G}_{2}-\mathrm{M}$ phases of the cell cycle was proportional to the amount of linear DAPI fluorescence, $G_{0}-G_{1}$ cells having half the amount of DAPI staining as the $\mathrm{S}-\mathrm{G}_{2}-\mathrm{M}$ cells.

Statistics. For all experiments with error bars, standard deviation was calculated to indicate the variation within each experiment. Mean differences between groups were examined by pooled Student's $t$ test (2-tailed). $P$ values less than 0.05 were considered statistically significant.

\section{Acknowledgments}

This study was supported by a Smith-Lemli-Opitz/RSH Foundation Research Grant (to M. He) and an SIMD/Ucyclyd fellowship grant (to M. He); and in part by NIH grant R01AG022379 (to A.N. Vallejo). J. Vockley was supported in part by NIH grant National Institute of Child Health and Human Development (NICHD) 1U54HD061939. This project was supported in part by the Sterol and Isoprenoid Diseases (STAIR) consortium, a part of the NIH Rare Diseases Clinical Research Network (RDCRN). Funding and/ or programmatic support for this project have been provided by U54HD061939 from the NICHD and the NIH Office of Rare Diseases Research (ORDR). The views expressed in written materials or publications do not necessarily reflect the official policies of the Department of Health and Human Services; nor does mention of trade names, commercial practices, or organizations imply endorsement by the U.S. government.

Received for publication February 12, 2010, and accepted in revised form December 8, 2010.

Address correspondence to: Jerry Vockley, Children's Hospital of Pittsburgh of UPMC, One Children's Hospital Drive, 4401 Penn Avenue, Pittsburgh, Pennsylvania 15224, USA. Phone: 412.692.7746; Fax: 412.692.7816; E-mail: gerard.vockley@chp.edu.

Lynne A. Wolfe's present address is: National Human Genome Research Institute, NIH, Bethesda, Maryland, USA.
1. Herman GE. Disorders of cholesterol biosynthesis: prototypic metabolic malformation syndromes. Hum Mol Genet. 2003;12 spec no. 1:R75-R88.

2. Rakheja D, Boriack RL. Precholesterol sterols accumulate in lipid rafts of patients with Smith-Lemli-Opitz syndrome and $\mathrm{X}$-linked dominant chondrodysplasia punctata. Pediatr Dev Pathol. 2008;11(2):128-132.

3. Janowski BA, Willy PJ, Devi TR, Falck JR, Mangelsdorf DJ. An oxysterol signalling pathway mediated by the nuclear receptor LXR alpha. Nature. 1996; 383(6602):728-731.

4. Castrillo A, et al. Crosstalk between LXR and tolllike receptor signaling mediates bacterial and viral antagonism of cholesterol metabolism. Mol Cell. 2003;12(4):805-816.

5. Shultz LD, et al. Mutations at the mouse ichthyosis locus are within the lamin B receptor gene: a single gene model for human Pelger-Huet anomaly. Hum Mol Genet. 2003;12(1):61-69.

6. Hoffmann K, et al. Mutations in the gene encoding the lamin $\mathrm{B}$ receptor produce an altered nuclear morphology in granulocytes (Pelger-Huet anomaly). Nat Genet. 2002;31(4):410-414.

7. Liu XY, et al. The gene mutated in bare patches and striated mice encodes a novel 3beta-hydroxysteroid dehydrogenase. Nat Genet. 1999;22(2):182-187.

8. Ishibashi M, Matsuda F, Oka H, Ishiko A. Abnormal lamellar granules in a case of CHILD syndrome. J Cutan Pathol. 2006;33(6):447-453.

9. Darnet S, Rahier A. Plant sterol biosynthesis: identification of two distinct families of sterol 4alphamethyl oxidases. Biochem J. 2004;378(pt 3):889-898.

10. Gachotte D, Pierson CA, Lees ND, Barbuch R, Koegel C, Bard M. A yeast sterol auxotroph (erg25) is rescued by addition of azole antifungals and reduced levels of heme. Proc Natl Acad SciU S A. 1997; 94(21):11173-11178.

11. Xie H, Xia G, Byskov AG, Andersen CY, Bo S, Tao Y. Roles of gonadotropins and meiosis-activating sterols in meiotic resumption of cultured follicle-enclosed mouse oocytes. Mol Cell Endocrinol. 2004;218(1-2):155-163.

12. Cukurcam $S$, et al. Influence of follicular fluid meiosis-activating sterol on aneuploidy rate and precocious chromatid segregation in aged mouse oocytes. Hum Reprod. 2007;22(3):815-828.

13. Gibson KM, et al. 3-Hydroxy-3-methylglutaryl coenzyme A reductase activity in cultured fibroblasts from patients with mevalonate kinase deficiency: differential response to lipid supplied by fetal bovine serum in tissue culture medium. J Lipid 
Res. 1990;31(3):515-521.

14. Hashimoto F, Hayashi H. Identification of intermediates after inhibition of cholesterol synthesis by aminotriazole treatment in vivo. Biochim Biophys Acta. 1991;1086(1):115-124.

15. Hashimoto F, Hayashi H. Peroxisomal cholesterol synthesis in vivo: accumulation of 4-methyl intermediate sterols after aminotriazole inhibition of cholesterol synthesis. Biochim Biophys Acta. 1994;1214(1):11-19.

16. Yilmaz A, et al. HMG-CoA reductase inhibitors suppress maturation of human dendritic cells: new implications for atherosclerosis. Atherosclerosis. 2004;172(1):85-93.

17. Begon E, et al. Expression, subcellular localization and cytokinic modulation of Toll-like receptors (TLRs) in normal human keratinocytes: TLR2 upregulation in psoriatic skin. Eur J Dermatol. 2007; 17(6):497-506

18. Candia L, Marquez J, Hernandez C, Zea AH, Espinoza LR. Toll-like receptor-2 expression is upregulated in antigen-presenting cells from patients with psoriatic arthritis: a pathogenic role for innate immunity? J Rheumatol. 2007;34(2):374-379.

19. Michel JJ, et al. CD56-expressing T cells that have features of senescence are expanded in rheumatoid arthritis. Arthritis Rheum. 2007;56(1):43-57.

20. Cho SG, et al. Psoriasiform eruption triggered by recombinant granulocyte-macrophage colony stimulating factor (rGM-CSF) and exacerbated by granulocyte colony stimulating factor (rG-CSF) in a patient with breast cancer. J Korean Med Sci. 1998; 13(6):685-688

21. Debets R, Hegmans JP, Deleuran M, Hooft S, Benner R, Prens EP. Expression of cytokines and their receptors by psoriatic fibroblast. I. Altered IL-6 synthesis. Cytokine. 1996;8(1):70-79.

22. Youssef S, et al. The HMG-CoA reductase inhibitor, atorvastatin, promotes a Th2 bias and reverses paralysis in central nervous system autoimmune disease. Nature. 2002;420(6911):78-84

23. Wassif CA, et al. Residual cholesterol synthesis and simvastatin induction of cholesterol synthesis in Smith-Lemli-Opitz syndrome fibroblasts. Mol
Genet Metab. 2005;85(2):96-107.

24. Jira PE, et al. Simvastatin. A new therapeutic approach for Smith-Lemli-Opitz syndrome. J Lipid Res. 2000;41(8):1339-1346.

25 . Bensinger SJ, et al. LXR signaling couples sterol metabolism to proliferation in the acquired immune response. Cell. 2008;134(1):97-111.

26 . Smyth I, et al. A mouse model of harlequin ichthyosis delineates a key role for Abca12 in lipid homeostasis. PLoS Genet. 2008;4(9):e1000192.

27. Darnet S, Rahier A. Enzymological properties of sterol-C4-methyl-oxidase of yeast sterol biosynthesis. Biochim Biophys Acta. 2003;1633(2):106-117.

28. Marijanovic $Z$, et al. Closing the gap: identification of human 3-ketosteroid reductase, the last unknown enzyme of mammalian cholesterol biosynthesis. Mol Endocrinol. 2003;17(9):1715-1725.

29. Kelley RI, Herman GE. Inborn errors of sterol biosynthesis. Annu Rev Genomics Hum Genet. 2001; 2:299-341.

30. Krakowiak PA, et al. Lathosterolosis: an inborn error of human and murine cholesterol synthesis due to lathosterol 5-desaturase deficiency. Hum Mol Genet. 2003;12(13):1631-1641

31. Grange DK, et al. Abnormal laterality development and proliferative epidermal skin lesions in $\mathrm{x}$-linked disorders of cholesterol biosynthesis. Proc Green wood Gen Center. 2001;20:105.

32. Simon A, et al. Mevalonate kinase deficiency: evidence for a phenotypic continuum. Neurology. 2004;62(6):994-997.

33. Kovarova M, Wassif CA, Odom S, Liao K, Porter FD, Rivera J. Cholesterol deficiency in a mouse model of Smith-Lemli-Opitz syndrome reveals increased mast cell responsiveness. J Exp Med. 2006; 203(5):1161-1171.

34. Mantha AJ, Hanson JE, Goss G, Lagarde AE, Lorimer IA, Dimitroulakos J. Targeting the mevalonate pathway inhibits the function of the epidermal growth factor receptor. Clin Cancer Res. 2005; 11(6):2398-2407.

35. Lajoie P, Nabi IR. Regulation of raft-dependent endocytosis. J Cell Mol Med. 2007;11(4):644-653

36 . Fontaine $\mathrm{C}$, et al. Liver $\mathrm{X}$ receptor activation poten- tiates the lipopolysaccharide response in human macrophages. Circ Res. 2007;101(1):40-49.

37. Hoffmann K, Sperling K, Olins AL, Olins DE. The granulocyte nucleus and lamin B receptor: avoiding the ovoid. Chromosoma. 2007;116(3):227-235.

38. Schon M, Denzer D, Kubitza RC, Ruzicka T, Schon MP. Critical role of neutrophils for the generation of psoriasiform skin lesions in flaky skin mice. J Invest Dermatol. 2000;114(5):976-983.

39. Kanekura T, Hiraishi K, Kawahara K, Maruyama I, Kanzaki T. Granulocyte and monocyte adsorption apheresis (GCAP) for refractory skin diseases caused by activated neutrophils and psoriatic arthritis: evidence that GCAP removes Mac-1-expressing neutrophils. Ther Apher Dial. 2006;10(3):247-256.

40. Greenwood J, Mason JC. Statins and the vascular endothelial inflammatory response. Trends Immunol. 2007;28(2):88-98.

41. Jury EC, Isenberg DA, Mauri C, Ehrenstein MR. Atorvastatin restores Lck expression and lipid raftassociated signaling in $\mathrm{T}$ cells from patients with systemic lupus erythematosus. J Immunol. 2006; 177(10):7416-7422.

42. Feingold KR, Man MQ, Menon GK, Cho SS, Brown $\mathrm{BE}$, Elias PM. Cholesterol synthesis is required for cutaneous barrier function in mice. J Clin Invest. 1990;86(5):1738-1745.

43. He $\mathrm{M}$, et al. A new genetic disorder in mitochondrial fatty acid beta-oxidation: ACAD9 deficiency. Am J Hum Genet. 2007;81(1):87-103.

44. Kelley RI. Diagnosis of Smith-Lemli-Opitz syndrome by gas chromatography/mass spectrometry of 7-dehydrocholesterol in plasma, amniotic fluid and cultured skin fibroblasts. Clin Chim Acta. 1995; 236(1):45-58

45. de Jager W, Prakken BJ, Bijlsma JW, Kuis W, Rijkers GT. Improved multiplex immunoassay performance in human plasma and synovial fluid following removal of interfering heterophilic antibodies. J Immunol Methods. 2005;300(1-2):124-135.

46. Pfleger C, Schloot N, ter Veld F. Effect of serum content and diluent selection on assay sensitivity and signal intensity in multiplex bead-based immunoassays. J Immunol Methods. 2008;329(1-2):214-218. 